\title{
Nanostructured Porous Silicon Photonic Crystal for Applications in the Infrared
}

\author{
G. Recio-Sánchez, V. Torres-Costa, M. Manso-Silván, and R. J. Martín-Palma \\ Departamento de Física Aplicada, Universidad Autónoma de Madrid, Avdenia Francisco Tomás y Valiente 7 , \\ Cantoblanco, 28049 Madrid, Spain \\ Correspondence should be addressed to G. Recio-Sánchez, gonzalo.recio@uam.es
}

Received 2 February 2012; Accepted 21 February 2012

Academic Editor: Omer Nur

Copyright ( 2012 G. Recio-Sánchez et al. This is an open access article distributed under the Creative Commons Attribution License, which permits unrestricted use, distribution, and reproduction in any medium, provided the original work is properly cited.

In the last decades great interest has been devoted to photonic crystals aiming at the creation of novel devices which can control light propagation. In the present work, two-dimensional (2D) and three-dimensional (3D) devices based on nanostructured porous silicon have been fabricated. $2 \mathrm{D}$ devices consist of a square mesh of $2 \mu \mathrm{m}$ wide porous silicon veins, leaving $5 \times 5 \mu \mathrm{m}$ square air holes. 3D structures share the same design although multilayer porous silicon veins are used instead, providing an additional degree of modulation. These devices are fabricated from porous silicon single layers (for 2D structures) or multilayers (for 3D structures), opening air holes in them by means of $1 \mathrm{KeV}$ argon ion bombardment through the appropriate copper grids. For 2D structures, a complete photonic band gap for TE polarization is found in the thermal infrared range. For 3D structures, there are no complete band gaps, although several new partial gaps do exist in different high-symmetry directions. The simulation results suggest that these structures are very promising candidates for the development of low-cost photonic devices for their use in the thermal infrared range.

\section{Introduction}

The concept of photonic crystal was proposed and discussed theoretically several decades ago $[1,2]$. Photonic crystals can be described as periodic dielectric structures exhibiting frequency ranges over which electromagnetic waves are not allowed to propagate. Lightwaves experience periodic perturbations when they propagate through these structures, analogous to electrons in a solid crystal [3-5]. This analogy suggests that the dispersion of electromagnetic waves in a photonic crystal can be described in terms of photonic band structures (PBSs). The frequency ranges over which electromagnetic waves propagation is not allowed are generally called photonic band gaps [6,7]. Thus, photonic crystals can control the propagation of light allowing novel applications in several fields, such as optical devices, including waveguides [8], lasers [9], or filters [10], or in the field of telecommunications as high-efficiency antenna substrates or reflec- tors [11]. The main handicap with photonic crystals is that they are not easy to fabricate and often require expensive semiconductor fabrication technology $[12,13]$.

In the present work, a relatively simple fabrication process is presented to manufacture $2 \mathrm{D}$ and $3 \mathrm{D}$ photonic crystals based on nanostructured porous silicon (PSi). A typical $2 \mathrm{D}$ structure consists of a square mesh of $2 \mu \mathrm{m}$ wide PSi veins, leaving $5 \mu \mathrm{m} \times 5 \mu \mathrm{m}$ holes of air. 3D structures are obtained from the same 2D structures, alternating PSi layers with different porosity leading to light modulation in an additional direction.

There are several reasons to choose PSi as the dielectric material in the photonic crystal. On the one hand, the refractive index of PSi can be controlled by simply changing the porosity of the layers [14]. In this regard, there are several models that relate the porosity of a PSi layer and its refractive index, such as the Maxwell-Garnett [15] or the Bruggeman models [16]. This last one is used in this work and relates 
porosity and effective refractive index through the following expression:

$$
p=1-\left[\frac{\left(1-n_{\mathrm{PSi}}^{2}\right)\left(n_{\mathrm{Si}}^{2}+2 n_{\mathrm{PSi}}^{2}\right)}{3 n_{\mathrm{PSi}}^{2}\left(1-n_{\mathrm{Si}}^{2}\right)}\right],
$$

where porosity $(p)$ is the percentage of air in a layer crosssection, $n_{\mathrm{Si}}$ is the refractive index of silicon, and $n_{\mathrm{PSi}}$ is the effective refractive index associated with that porosity.

On the other hand, porous silicon is a very suitable material for the fabrication of photonic devices thanks to the ease of integration in silicon technology [17]. Its efficient photoluminescence in the visible range at room temperature $[18,19]$, or the possibility to turn PSi into a biocompatible material, makes PSi a very interesting material to generate novel applications including high-efficiency biosensing platforms [20-22].

\section{Fabrication of the Photonic Crystals}

The $2 \mathrm{D}$ periodic dielectric structures that have been fabricated consist of a square mesh of $2 \mu \mathrm{m}$ wide porous silicon veins, leaving $5 \times 5 \mu \mathrm{m}$ square air holes. $3 \mathrm{D}$ structures share the same design although multilayer porous silicon veins are used instead, providing an additional degree of modulation.

To fabricate the photonic structure, several steps are needed. First, PSi layers (2D) or multilayers (3D) are grown on a silicon wafer. PSi is grown by electrochemical etching crystalline silicon wafers in hydrofluoric acid solutions. Several parameters are important in this process, which determines the properties of the PSi layer. The most significant parameters are the applied current density, HF concentration in the solution, and etching time. By changing these parameters it is possible to control the porosity and the thickness of the PSi layers. To fabricate a multilayer structure, the applied current density is varied during the etching process. Further details are given in $[23,24]$.

Once the PSi layer (or multilayer for 3D) is formed, a Cu grid is placed on top of the device in contact with the surface of PSi. The Cu grids used were 2000 Gilder TEM Cu mesh, with a structure of square veins $2 \mu \mathrm{m}$ wide, leaving air holes of $5 \mu \mathrm{m} \times 5 \mu \mathrm{m}$, and a thickness of $20 \mu \mathrm{m}$. Then a $1 \mathrm{KeV}$ Argon ion bombardment is performed using a Kaufman source, to open holes of air in the PSi matrix. After the bombardment, the $\mathrm{Cu}$ grid is removed, as is shown in Figure 1.

In Figure 2, a SEM image of a typical 3D photonic structure is presented. The structure consists of 20 periods of two different Psi layers of the same thickness. Each period was formed by varying the applied current density between $10 \mathrm{~mA} / \mathrm{cm}^{2}$ for $30 \mathrm{~s}$ (leading to a layer of $40 \%$ porosity and 0.5 microns of thickness) and $150 \mathrm{~mA} / \mathrm{cm}^{2}$ for $5 \mathrm{~s}$ (leading to a layer of $80 \%$ porosity and 0.5 microns of thickness). It can be observed that after the ion bombardment process square air holes are opened in the PSi multilayer matrix stack although only around 7 periods have been etched.

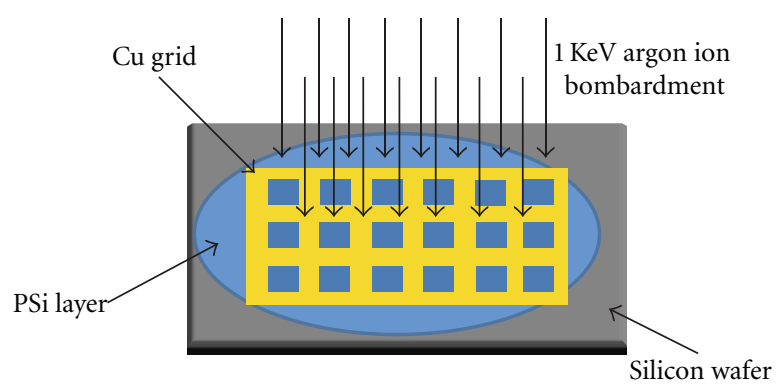

FIgURE 1: Schematic representation of the photonic crystal structure fabrication process.

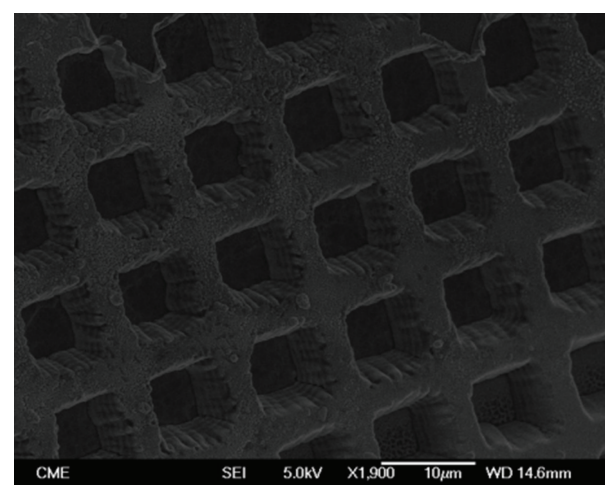

Figure 2: Scanning electron microscope image of a 3D photonic structure made by the new fabrication process shown.

\section{Determination of the Photonic Band Structure}

The photonic band structure of 2D and 3D PSi-based photonic crystals was computed. In order to determine the PBS, the MIT Photonic Bands (MPB) package [25] was used. This software is based on the plane-wave expansion method.

Transmittance response in the high-symmetry directions was calculated using the Translight software [26], based on the matrix transfer method.

3.1. 2D Photonic Crystals. The dielectric structure used to calculate 2D PBS was a square grid of dielectric veins in air, with a width of $0.285 a, a$ being the lattice parameter, as shown in the inset of Figure 3. The dielectric constant of the PSi veins was set as a function of the porosity of PSi (see (1)), being $\sqrt{\varepsilon}=n_{\mathrm{PSi}}$.

Figure 3 shows the PBS of a 2D structure based on PSi layer with a $40 \%$ of porosity $(\varepsilon=4.84)$. A complete band gap for TE polarization between 0.35 and 0.375 normalized frequency can be clearly observed. Also, there are several partial gaps in the $\Gamma-\mathrm{X}$ and $\Gamma$-M high-symmetry directions for both TE and TM polarization modes.

The position and shape of the gaps of these structures can be tuned by changing either the pattern of the dielectric veins or their dielectric constant (i.e., porosity of the PSi layers). The pattern design is limited, in this case, to the available grid designs. However, the dielectric constant of the PSi layers is 


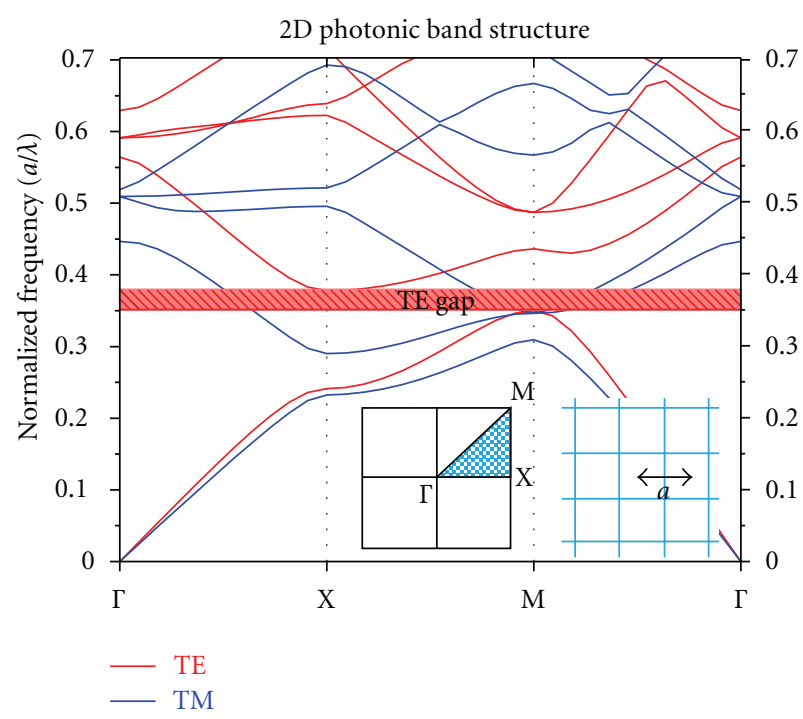

FIGURE 3: 2D calculated PBS for a structure based on a square mesh of dielectric veins with a width of $0.285 a$, with the dielectric material being a PSi layer with $40 \%$ porosity $(\varepsilon=4.84)$. Bands are plotted along high-symmetry lines of the Brillouin zone, where frequency is given in normalized units $(a / \lambda)$. The representation of $2 \mathrm{D}$ Brillouin zone and the schematic representation of $2 \mathrm{D}$ structure used for the calculations are shown in the inset of PBS.

simply adjustable by changing the main parameters in the PSi fabrication process.

Figure 4 shows the TE complete gap map for all possible PSi porosity values, from which it is observed that porosity has a strong influence on the size and frequency of the photonic band gaps. It can be also noted that the gap does not exist for all the porosity ranges. For porosities higher than $50 \%$, the gap disappears. Above this porosity, the effective refractive index of the PSi layer is lower than 1.5. Thus, the contrast between the refractive indexes of the two media which form the photonic device (air and PSi) is not high enough to perturb the electromagnetic waves. In this case, the TE complete gap does not appear.

Moreover, the lower the porosity, the larger the size of the gap. This is an expected behavior since the lower the porosity, the larger the refractive index associated to the PSi layer, and consequently, the greater the contrast between refractive indexes.

In Figure 4, the relationship between the porosity of PSi layer and their effective refractive index, according to (1), is indicated on the top $x$ axis. Also, the equivalent wavelength is shown on the right $y$ axis, for lattice parameter $a=7 \mu \mathrm{m}$, the same as the experimental one (see Section 2). It can be observed that the gap appears in the thermal infrared region, between 17 and $27 \mu \mathrm{m}$, depending on the porosity of the PSi layers.

Figures 5(a) and 5(b) show the partial gap maps in two different high-symmetry directions, $\Gamma-\mathrm{X}$ and $\Gamma-\mathrm{M}$, respectively. In these figures, several gaps in those directions for both polarization modes are shown, almost the whole porosity range. In this way, by selecting the appropriate porosity

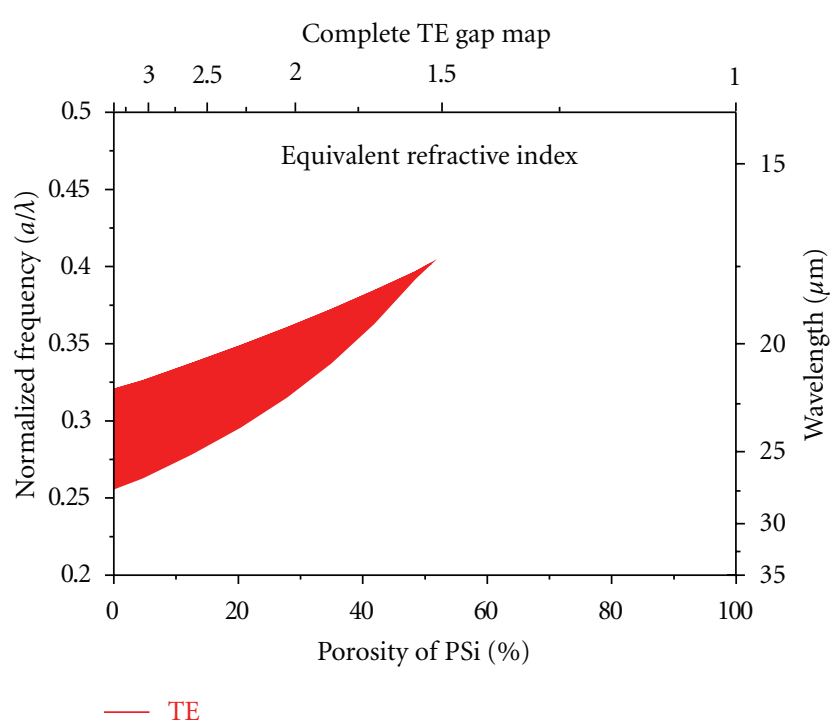

Figure 4: Complete TE gap map as a function of the PSi layer porosity. In the right $y$ axis, equivalent wavelength is indicated for a lattice parameter of $a=7 \mu \mathrm{m}$, the same as the experimental one (see Section 3). In the top $x$ axis, effective refractive index is shown following (1).

for the PSi layers, the size and frequency position of the partial gaps can be chosen for a given application. To determine the wavelength, indicated on the right $y$ axis, the lattice parameter was set $a=7 \mu \mathrm{m}$, as in Figure 4. In Figure 5(a) it can be observed that the wavelength range where the partial $\Gamma-\mathrm{X}$ gaps appear is quite broad, extending from $40 \mu \mathrm{m}$ to a few $\mu \mathrm{m}$ for both polarization modes. That enables the control of light propagation in that direction of the slab in a large frequency range.

In Figure 5(b), the partial $\Gamma-\mathrm{M}$ gap map shows a similar range of frequencies for the directional gap, ranging from almost $30 \mu \mathrm{m}$ to a few microns, allowing the possibility of tuning electromagnetic waves in other preferential direction, for the same 2D structure.

Next, transmittance in the high-symmetry directions was computed by using the Translight software, considering a square mesh of air columns in a dielectric medium, with an area of $0.715 a \times 0.715 a$ and a mesh cell of $128 \times 128$. In Figure 6, the TM transmittance of a structure with a PSi layer of $40 \%$ porosity $(\varepsilon=4.84)$ in the $\Gamma$-X direction is compared with its computed PBS. A good agreement between the two simulation methods is observed. It should be noticed that at the frequencies where the partial gap appears, transmittance is zero. This is an expected result since at the frequencies where the gap opens, the light cannot propagate through the device; thus, the transmittance will be zero, being the reflectance maximum.

As it can be observed in Figure 6, transmittance is zero between the frequency ranges 0.25 and $0.32,0.5$ and 0.54 , and around 0.57 and 0.78 , where the partial gaps appear.

3.2. 3D Photonic Crystals. 3D photonic structures are fabricated following the same procedure as the $2 \mathrm{D}$ structures, 


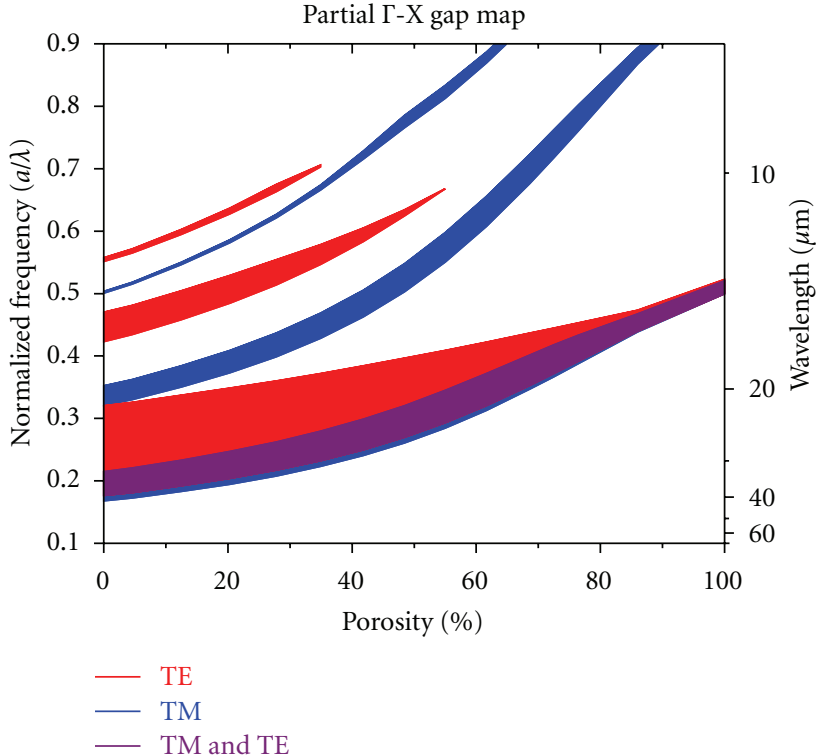

(a)

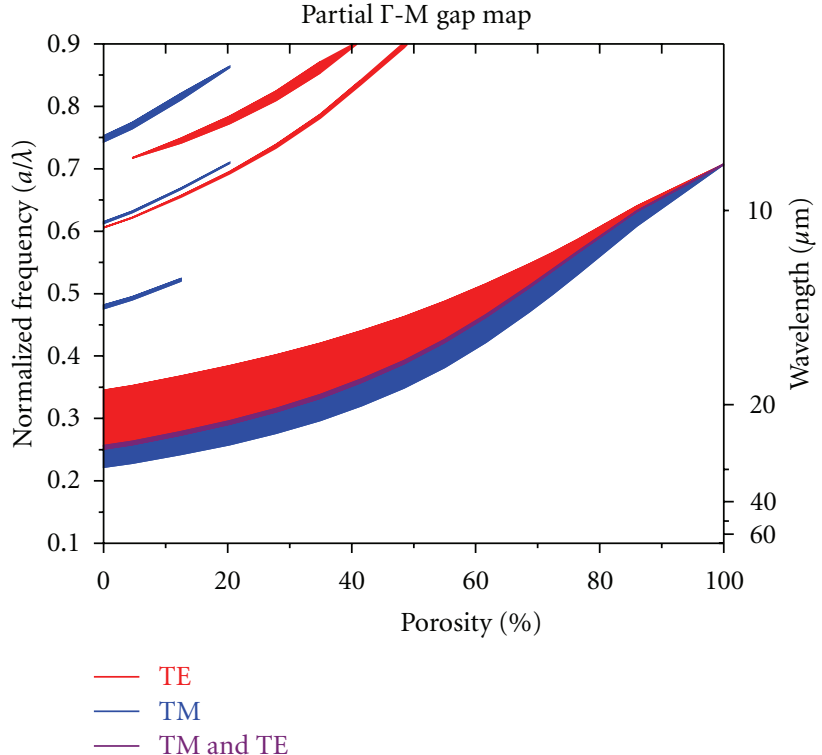

(b)

FIgure 5: (a) Partial $\Gamma$-X gap map as a function of the PSi layer porosity. On the right $y$ axis, equivalent wavelength is indicated for a lattice parameter $a=7 \mu \mathrm{m}$. (b) Partial $\Gamma$-M gap map as a function of the PSi layer porosity. The wavelength on right $y$ axis is indicated for a lattice parameter $a=7 \mu \mathrm{m}$, as the experimental one.

$\Gamma$-X direction

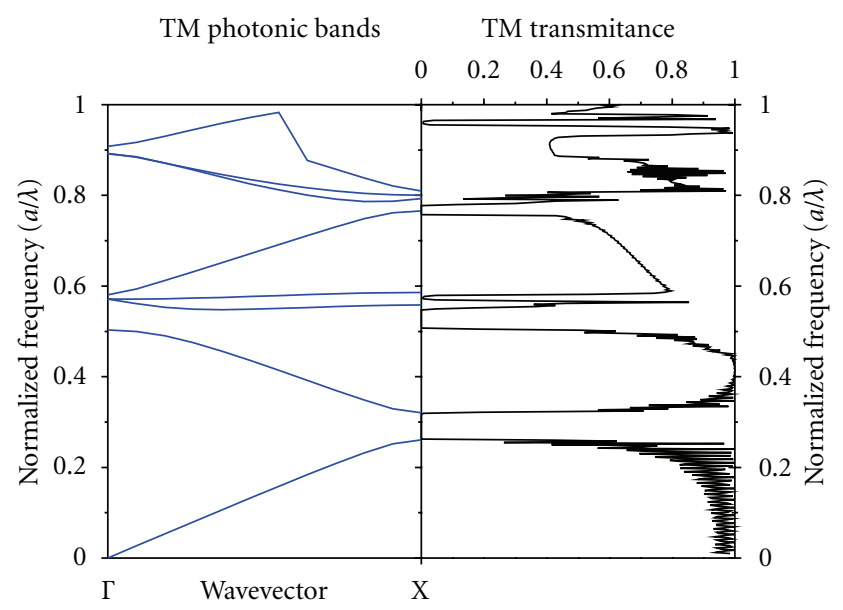

Figure 6: On the left, TM bands along the $\Gamma-\mathrm{X}$ direction for a 2D structure with a PSi layer of $40 \%$ porosity $(\varepsilon=4.84)$ are represented. On the right pane, TM transmittance response for the same structure in the same direction is shown. In both graphs, the frequency is given in normalized units $(a / \lambda)$.

but fabricating PSi multilayers, consisting in alternating PSi layers with different porosities, as represented in the inset of Figure 7.

Figure 7 shows the PBS of a typical 3D photonic structure. The PSi multilayer is based on alternating PSi layers of 40 and $80 \%$ porosity $(\varepsilon=4.84$ and $\varepsilon=1.96)$, and a thickness of $0.5 a$ each, while the width of the square mesh is kept to $0.285 a$. It can be appreciated that there are no complete gaps, although several partial gaps in the high-symmetry

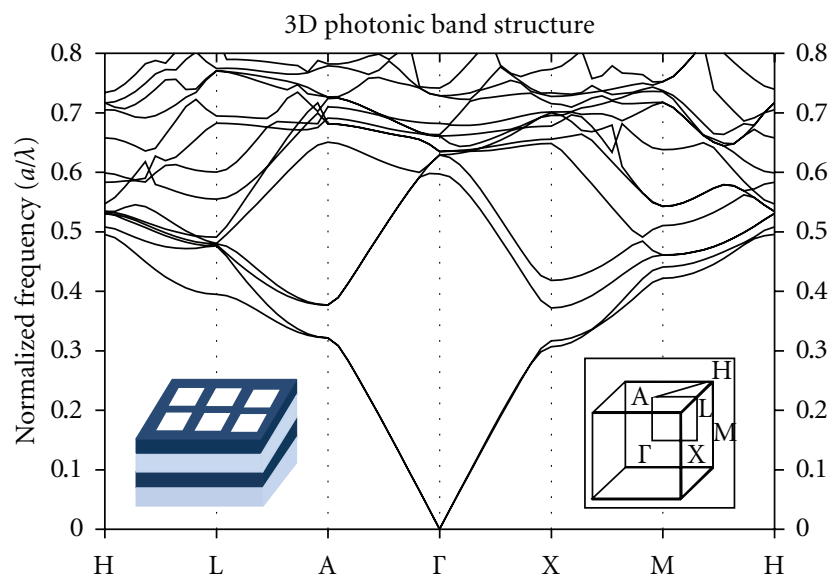

Figure 7: 3D calculated PBS for a 3D structure based on alternating Psi layers of 40 and $80 \%$ porosity and $0.5 a$ thickness each. Bands are plotted along main symmetry lines of the Brillouin zone, where frequency is given in normalized units $(a / \lambda)$. Left inset, the schematic representation of $3 \mathrm{D}$ structure used in the simulations is represented. In dark blue the low-porosity Psi layers and in light blue the high-porosity Psi layers are shown. Right inset, the representation of 3D Brillouin zone is shown.

directions do exist. Firstly, it has to be noticed that the partial gaps in the $\Gamma-\mathrm{X}$ and $\Gamma-\mathrm{M}$ directions (the last one is not shown in Figure 7) still exist in the 3D structure. The size and frequency of these partial gaps depend on the porosity of the PSi layers used in the multilayer stack. Therefore, new partial gaps appear in the new high-symmetry directions $\Gamma$-A and $\Gamma-\mathrm{L}$ (this one neither appears in Figure 7). The size and frequency of these new gaps can be modified by changing 


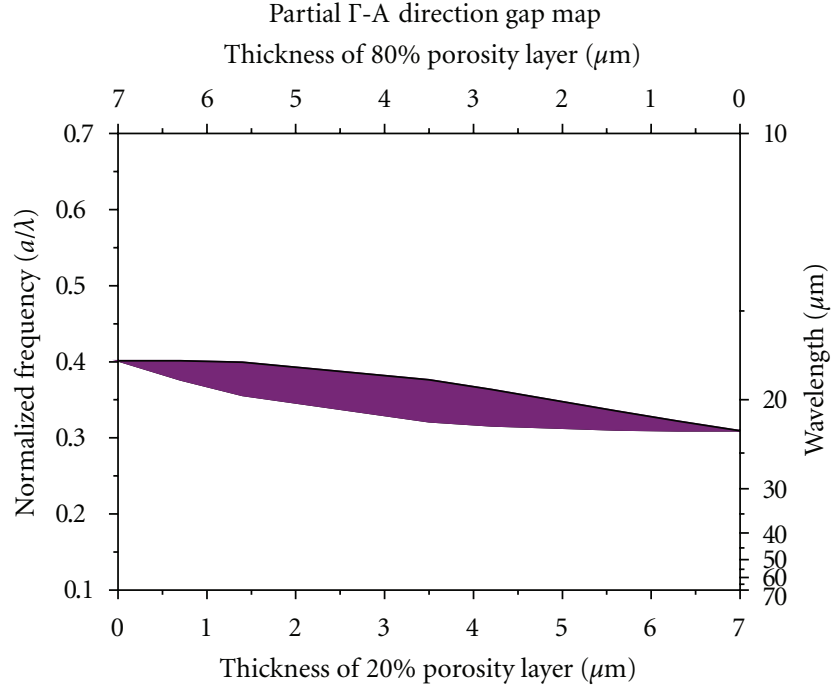

Figure 8: Partial $\Gamma$-A gap map as a function of the thickness of the different PSi layers. The lattice parameter used was $a=7 \mu \mathrm{m}$, as the experimental one (see Section 2).

the porosity of the multilayer used, in the same way as it was made in the case of the $2 \mathrm{D}$ partial gaps. However, now there is another way to tune these new gaps, by changing the thickness of the different PSi layers.

Figure 8 shows the partial gap map in $\Gamma$-A directions, respectively, as a function of the thickness of the different PSi layers used to fabricate the unit cell, using 7 microns as the lattice parameter. It can be observed that the widest gaps appear when the thickness of the two different PSi layers is similar. Moreover, if the unit cell is formed by only one kind of PSi layer, the new gap disappears.

\section{Conclusions}

A novel and simple fabrication process of 2D and 3D photonic crystal based on nanostructured porous silicon, to operate in the thermal infrared region, is presented. 2D slabs, based on a square mesh of $2 \mu \mathrm{m}$ wide dielectric veins leaving $5 \mu \mathrm{m} \times 5 \mu \mathrm{m}$ air holes, show a complete gap for the TE polarization mode. Also partial gaps in the high-symmetry $\Gamma-\mathrm{X}$ and $\Gamma-\mathrm{M}$ directions appear for both TE and TM polarization modes. The size and frequency position of the gaps can be controlled by adjusting the porosity of the PSi layer which allows fabricating photonic crystal devices for specific applications. Complete TE gap appears over a frequency range between 27 and $17 \mu \mathrm{m}$, whereas $\Gamma-\mathrm{X}$ and $\Gamma-\mathrm{M}$ partial gaps open from $40 \mu \mathrm{m}$ and from $30 \mu \mathrm{m}$ to a few microns, respectively.

$3 \mathrm{D}$ photonic structures are based on the $2 \mathrm{D}$ structures, but alternating PSi layers with different porosity. The 3D structures exhibit new partial gaps in the new high-symmetry directions $\Gamma-\mathrm{A}$ and $\Gamma-\mathrm{L}$. These new partial gaps can be tuned by changing the porosity of the PSi layers used, in the same way as the 2D structures, but also it is possible to control these new gaps by modifying the thickness of the individual PSi layers. The theoretical results suggest that these structures could be very promising candidates to develop low-cost silicon-based photonic crystal devices to operate in the thermal infrared region.

\section{Acknowledgments}

The authors thank L. García-Pelayo for technical support in the realization of the present work and acknowledge Centro de Computación Científica (Universidad Autónoma de Madrid) for providing computational resources for numerical calculations. The authors also gratefully acknowledge funding from Comunidad de Madrid (Spain) under project "Microseres" and Ministerio de Economía y Competitividad (Spain) under Research Project MAT2011-28345-C02-01.

\section{References}

[1] K. Ohtaka, "Energy band of photons and low-energy photon diffraction," Physical Review B, vol. 19, no. 10, pp. 5057-5067, 1979.

[2] E. Yablonovitch, "Inhibited spontaneous emission in solidstate physics and electronics," Physical Review Letters, vol. 58, no. 20, pp. 2059-2062, 1987.

[3] J. D. Joannopoulos, Photonic Crystals: Molding the Flow of Light, Princeton University Press, Princeton, NJ, USA, 2008.

[4] K. Sakoda, Optical Properties of Photonic Crystals, Springer Verlag, New York, NY, USA, 2005.

[5] S. John, "Strong localization of photons in certain disordered dielectric superlattices," Physical Review Letters, vol. 58, no. 23, pp. 2486-2489, 1987.

[6] K. M. Ho, C. T. Chan, and C. M. Soukoulis, "Existence of a photonic gap in periodic dielectric structures," Physical Review Letters, vol. 65, no. 25, pp. 3152-3155, 1990.

[7] E. Yablonovitch, "Photonic band-gap structures," Journal of the Optical Society of America B, vol. 10, no. 2, pp. 283-295, 1993.

[8] J. S. Foresi, P. R. Villeneuve, J. Ferrera et al., "Photonic-bandgap microcavities in optical waveguides," Nature, vol. 390, no. 6656, pp. 143-145, 1997.

[9] B. Temelkuran, S. D. Hart, G. Benoit, J. D. Joannopoulos, and Y. Fink, "Wavelength-scalable hollow optical fibres with large photonic bandgaps for CO2 laser transmission," Nature, vol. 420, no. 6916, pp. 650-653, 2002.

[10] M. Qiu and B. Jaskorzynska, "Design of a channel drop filter in a two-dimensional triangular photonic crystal," Applied Physics Letters, vol. 83, no. 6, pp. 1074-1076, 2003.

[11] M. Thèvenot, C. Cheype, A. Reineix, and B. Jecko, "Directive photonic-bandgap antennas," IEEE Transactions on Microwave Theory and Techniques, vol. 47, no. 11, pp. 2115-2122, 1999.

[12] C. C. Cheng and A. Scherer, "Fabrication of photonic bandgap crystals," Journal of Vacuum Science and Technology B, vol. 13, no. 6, pp. 2696-2700, 1995.

[13] M. Campbell, D. N. Sharp, M. T. Harrison, R. G. Denning, and A. J. Turberfield, "Fabrication of photonic crystals for the visible spectrum by holographic lithography," Nature, vol. 404, no. 6773 , pp. 53-56, 2000.

[14] C. Pickering, M. I. J. Beale, D. J. Robbins, P. J. Pearson, and R. Greef, "Optical properties of porous silicon films," Thin Solid Films, vol. 125, no. 1-2, pp. 157-163, 1985.

[15] J. Garnett, "Colours in metal glasses and in metallic films," Philosophical Transactions of the Royal Society of London A, vol. 203, pp. 385-420, 1904. 
[16] D. A. G. Bruggeman, "Berechnung verschiedener physikalischer konstanten von heterogenen substanzen. I. Dielektrizitatskonstanten und leitfahigkeiten der mischkorper aus isotropen substanzen," Annalen der Physik, vol. 416, no. 7, pp. 636-664, 1935.

[17] K. D. Hirschman, L. Tsybeskov, S. P. Duttagupta, and P. M. Fauchet, "Silicon-based visible light-emitting devices integrated into microelectronic circuits," Nature, vol. 384, no. 6607, pp. 338-341, 1996.

[18] L. T. Canham, "Silicon quantum wire array fabrication by electrochemical and chemical dissolution of wafers," Applied Physics Letters, vol. 57, no. 10, pp. 1046-1048, 1990.

[19] M. Makarova, J. Vuckovic, H. Sanda, and Y. Nishi, "Twodimensional porous silicon photonic crystal light emitters," in Proceedings of the Conference on Lasers and Electro-Optics and 2006 Quantum Electronics and Laser Science Conference (CLEO/QELS '06), May 2006.

[20] F. Cunin, T. A. Schmedake, J. R. Link et al., "Biomolecular screening with encoded porous-silicon photonic crystals," $\mathrm{Na}$ ture Materials, vol. 1, no. 1, pp. 39-41, 2002.

[21] T. Jalkanen, V. Torres-Costa, J. Salonen et al., "Optical gas sensing properties of thermally hydrocarbonized porous silicon Bragg reflectors," Optics Express, vol. 17, no. 7, pp. 5446-5456, 2009.

[22] R. J. Martín-Palma, M. Manso, M. Arroyo-Hernández, V. Torres-Costa, and J. M. Martínez-Duart, "Nanostructuredporous-silicon-based two-dimensional photonic crystals," Applied Physics Letters, vol. 89, no. 5, Article ID 053126, 2006.

[23] V. Torres-Costa, R. J. Martín-Palma, and J. M. MartínezDuart, "Optical characterization of porous silicon films and multilayer filters," Applied Physics A, vol. 79, no. 8, pp. 1919 1923, 2004.

[24] R. J. Martín-Palma, V. Torres-Costa, M. Arroyo-Hernández, M. Manso, J. Pérez-Rigueiro, and J. M. Martínez-Duart, "Porous silicon multilayer stacks for optical biosensing applications," Microelectronics Journal, vol. 35, no. 1, pp. 45-48, 2004.

[25] S. G. Johnson and J. D. Joannopoulos, "Block-iterative frequency-domain methods for Maxwell's equations in a planewave basis," Optics Express, vol. 8, no. 3, pp. 173-190, 2001.

[26] A. Reynold, Translight Software, University of Glasgow, Glasgow, UK, 2000. 

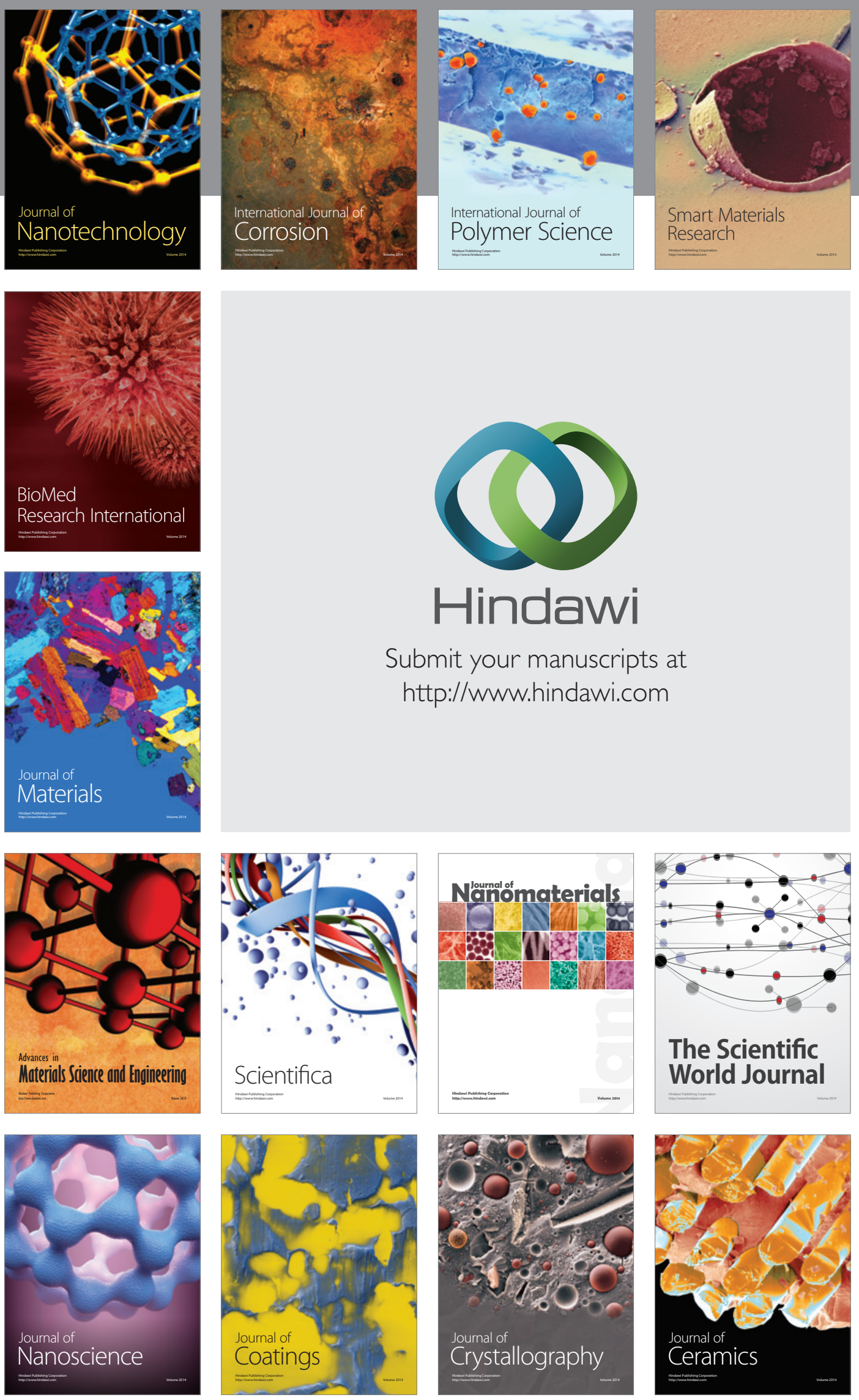

The Scientific World Journal

Submit your manuscripts at

http://www.hindawi.com

\section{World Journal}

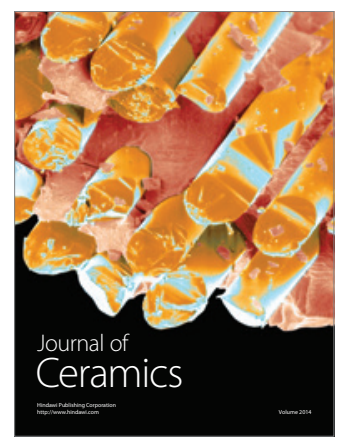

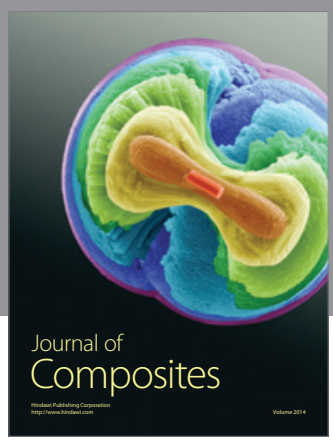
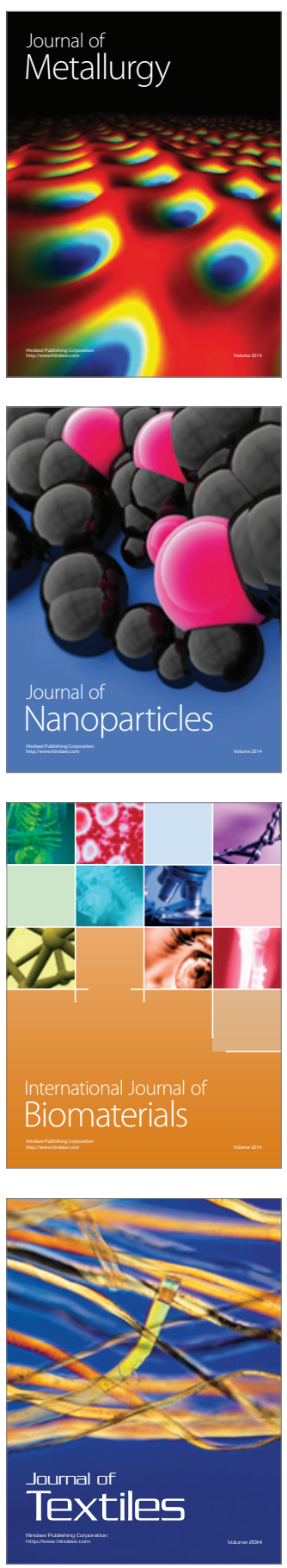